Reprod. Nutr. Dévelop., 1981, 21 (5A), 737-748.

\title{
Détermination du profil alimentaire de l'Oie
}

\section{de race landaise}

par J. C. MARCILLOUX, P. AUFFRAY

avec la collaboration technique de L. ROYER

Laboratoire de Physiologie de la Nufrition, INRA, 78350 Jouy en Josas, France.

Summary. Feeding pattern of the Landes goose.

This is a study of the comparative physiology of the feeding sequence in 24 Landes geese fed ad libitum. The relative parameters of feeding behavior and the apparatuses used have been described. We found that the 24-hour light/dark cycle, corresponding to maximal ingestion, was not the same in all the animals studied. Two groups were distinguished : those tending to be diurnal and those tending to be nocturnal. The feeding pattern of the animals in each group and from day to day was constant. The correlation between the amount ingested during the meal and the length of pre or post-prandial intervals showed great variation, and the choice of meal definition considerably affected the results.

\section{Introduction.}

Chez diverses espèces, mais essentiellement chez le Rat, la mise au point d'appareillages réalisant l'enregistrement continu et automatique des consommations a permis l'étude partielle ou complète des diverses caractéristiques de la périodicité alimentaire. Chez les Oiseaux les données en ce domaine sont encore rares ; on peut citer : Van Hemel et Myer (1969) chez la Caille, Zeigler et al. (1971) chez le Pigeon, Duncan et al. (1970) ainsi que Goussopoulos et al. (1973) et Clifton (1979) chez le Poulet, qui rapportent le rythme circadien des consommations de ces animaux. La séquence alimentaire de l'Oie n'a fait jusqu'à ce jour l'objet d'aucune analyse, c'est pourquoi nous avons été amenés à concevoir et réaliser des dispositifs permettant l'enregistrement graphique continu de sa prise de nourriture. Notre étude entre dans le cadre des travaux d'Auffray et Blum (1970), Auffray ef al. (1973) relatifs à la recherche d'une technique nouvelle de production de foie gras par la méthode dite d'hyperphagie provoquée. Ces auteurs obtiennent par lésion ou injection de 6-hydroxydopamine au niveau du noyau ventromédian hypothalamique de l'Oie une surconsommation d'aliment engendrant obésité et stéatose hépatique. L'obstacle majeur qui s'oppose à l'application industrielle de cette méthode est le manque de compétitiviłé quant aux poids des foies gras obtenus par ce procédé en comparaison de ceux obłenus lors du gavage traditionnel. Sachant que les rythmes d'ingestion influencent largement le degré d'obé- 
sité, il semble important de connaître les particularités du comportement alimentaire de l'Oie normale afin qu'ultérieurement on puisse envisager d'intervenir sur celui-ci pour tenter d'accroître le niveau d'obésité et espérer ainsi améliorer le poids des foies.

\section{Matériel et méthode.}

1) Dispositifs d'enregistrement.

a) Dispositif $n^{0} 1$. - Cet appareil ẹst schématisé sur la figure 1. L'appareillage est composé d'un disque horizontal sur lequel sont réparties des mangeoires selon des

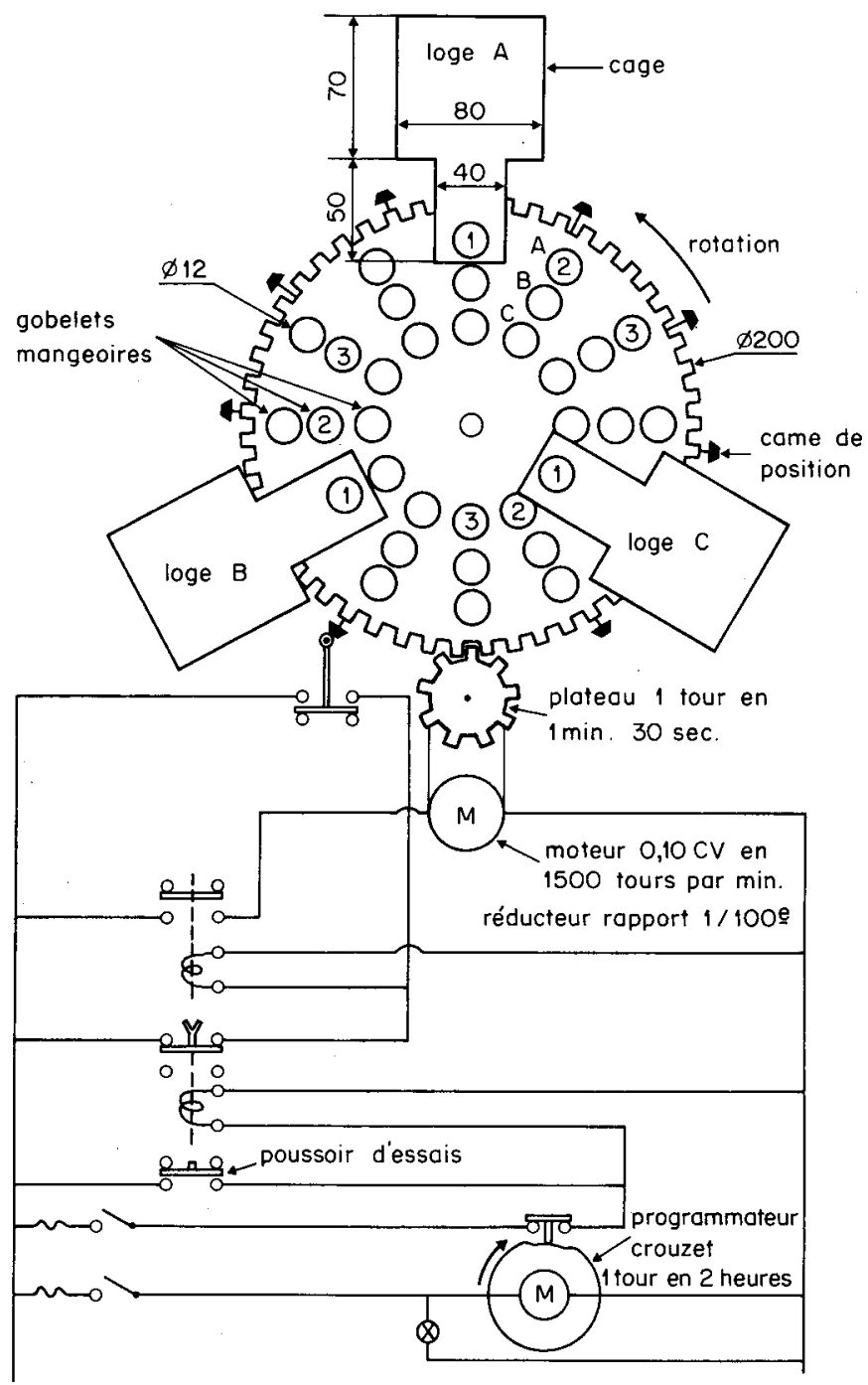

FIG. 1. - Dispositif de distribution automatique d'aliment destiné à l'étude du comportement alimentaire de l'Oie (dimensions données en cm). 
rangées concentriques. Un programmateur commande le moteur qui assure la rotation du disque. Des cages individuelles disposées autour de ce dispositif ne permettent l'accès d'un animal donné, qu'à une seule mangeoire. Un faisceau lumineux traverse deux orifices pratiquées à la partie supérieure de chaque mangeoire et vient frapper une cellule photoélectrique reliée à un enregistreur. Lorsque l'Oie effectue une prise d'aliment, elle provoque la rupture du faisceau lumineux, ce qui permet par inscription sur le papier de l'enregistreur se déroulant à la vitesse de $240 \mathrm{~mm}$ à l'heure, de connaître le nombre des repas ayec leur durée ef le moment précis où ils sont effectués ainsi que les intervalles qui les séparent. Les quantités ingérées sont mesurées par pesée des refus. Nous disposions, pour l'installation du dispositif d'enregistrement, d'un local d'une surface de $16 \mathrm{~m}^{2}$. Compte tenu de cet impératif, nous avons réalisé un appareillage permettant l'étude simultanée du comportement alimentaire de trois Oies à l'aide de 12 mangeoires par animal.

L'inconvénient majeur de ce système réside dans le fait que nous ne pouvons connaître les quantités ingérées par repas, mais uniquement celles des valeurs cumulées pendant le temps de programmation choisi. D'autre part, il existe un risque de déclenchement du programmateur, à l'issue du temps choisi, provoquant la rotation du disque au moment où l'Oie consomme. Aussi, avons-nous modifié la commande de l'appareillage de façon telle qu'un changement de mangeoire soit effectué après chaque repas.

b) Description de l'appareillage modifié (dispositif $n^{\circ} 2$ ). - Les passages successifs de la tête de l'animal au sein de sa mangeoire produisent, par coupure du faisceau lumineux frappant la cellule photoélectrique, un train d'impulsions. Ces impulsions utilisées par un montage électronique original provoquent le déclenchement du système assurant la rotation du disque après chaque repas. Le montage a été conçu selon divers critères établis grâce à l'analyse des données apportées par le dispositif initial. Dans ce dispositif un circuit d'interdiction, commandé par la cellule photoélectrique, empêche la rotation du plateau quand l'Oie effectue un repas.

Le critère repas étant l'élément essentiel de la séquence alimentaire, nous avons été amenés à en donner une définition.

c) Définition du repas. - Nous avons estimé, après observation des premiers tracés d'enregistrement, qu'un repas commençait lorsque l'animal se présentait au moins trois fois à sa mangeoire dans un laps de temps ne dépassant pas $20 \mathrm{sec}$. D'autre part, à titre de convention, nous avons considéré que deux prises alimentaires espacées de moins de $7 \mathrm{~min} 30 \mathrm{sec}$ font partie d'un même repas.

Le montage répond donc aux impératifs suivants : Si l'animal vient au moins trois fois en moins de 20 sec à sa mangeoire, une minuterie s'arme pour un temps de $7 \mathrm{~min} 30 \mathrm{sec}$. Chaque prise alimentaire nouvelle survenant dans ce laps de temps réarme celle-ci pour une durée identique. Si aucune autre venue n'est enregistrée, le repas est considéré comme terminé et le plateau distributeur tournera à l'issue des $7 \mathrm{~min} 30 \mathrm{sec}$. Cet appareillage n'admet qu'un seul animal à la fois et l'étude s'effectue à l'aide de 36 mangeoires. La quantité ingérée sera connue par pesée du refus. La figure 2 montre le synoptique de ce système. Il est à noter que ces deux dispositifs présentent la nourriture à l'animal sans aucune contrainte de ce dernier. L'accès à la mangeoire est libre, ce qui évite les artéfacts venant d'un éventuel effort pour consommer ou bien provoqués par un phénomène de conditionnement. 


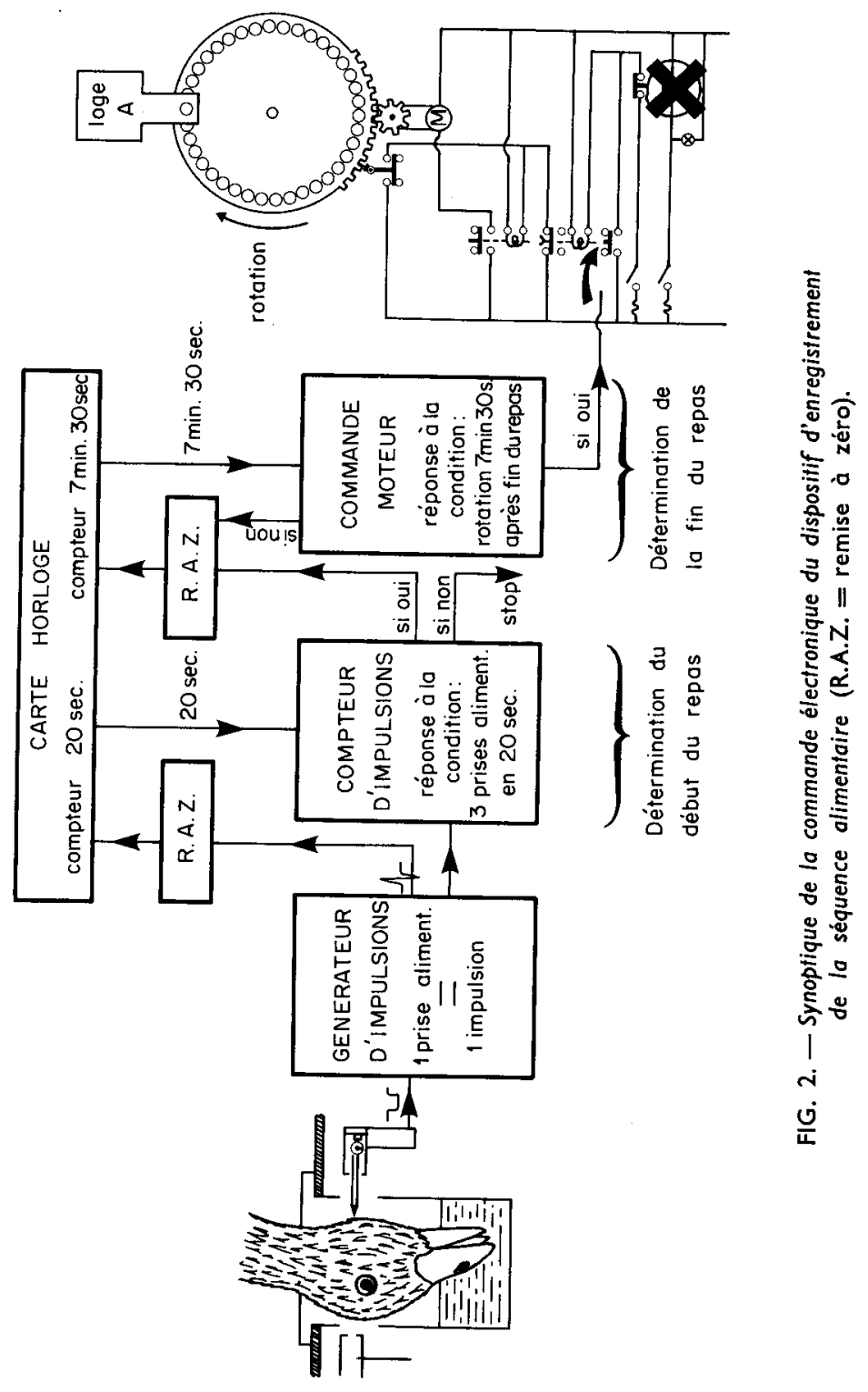




\section{Conditions expérimentales.}

La séquence alimentaire de 24 Oies de race landaise, adultes, de 5 à 6 mois d'âge et d'un poids moyen de $5,5 \pm 0,2 \mathrm{~kg}$ a été enregistrée. Le lot d'animaux était composé de 17 mâles et de 7 femelles, sexes déterminés à postériori lors de l'abattage.

Les animaux sont soumis à une période d'adaptation d'une dizaine de jours dans leurs cages, ces dernières étant placées dans une pièce où la température oscille entre 18 et 20 C. Les animaux ne sont isolés ni visuellement ni phonétiquement les uns des autres. L'éclairage est assuré par des fubes fluorescents, type lumière du jour, produisant une intensité lumineuse de 1000 lux au niveau de l'animal ; une minuterie assure une photopériode constante. Le cycle lumineux 12/12 est maintenu pendant la durée de l'expérience, l'éclairement étant assuré de $9 \mathrm{~h}$ à 21 h. Au cours de cette période les Oies reçoivent ad libitum, sous forme semi liquide (une partie de farine pour deux parties d'eau), un régime haute énergie comportant 47 p. 100 d'amidon, 18 p. 100 de protéines et 9 p. 100 de lipides. Ce régime apporte 4742 calories d'énergie brute par $\mathrm{kg}$ de matière sèche.

Composition du régime : Maïs : 74 p. 100, tourteau de soja : 15 p. 100 , farine de poisson : 3 p. 100, suif : 5 p. 100, calcimarine : 0,5 p. 100 , chlorure de sodium : 0,5 p. 100 , complément vitaminique : 1,5 p. 100, oligo-éléments : 0,5 p. 100 .

La pesée des refus alimentaires est effectuée après séchage à l'étuve ventilée à $80^{\circ} \mathrm{C}$ pendant une nuit. L'eau de boisson est distribuée ad libifum. Nous n'avons pas évalué les consom mations car le gaspillage de foilettage représente une source d'erreur trop importante pour accorder une quelconque valeur aux mesures éventuelles.

Les résultałs sont analysés à l'aide du test statistique de Student (test t) (Snedecor et Cochran, 1971).

Nous avons défini le profil alimentaire à l'aide des paramètres suivants : 1) Consommation journalière d'aliment avec étude de la répartition temporelle de celle-ci ; 2) Nombre de repas effectués par 24 h ; 3) Durée moyenne de ces repas ; 4) Quantité moyenne ingérée au cours de chacun de ces repas; 5) Vitesse d'ingestion ; 6) Durée moyenne d'un intervalle entre deux repas.

Nous rapportons dans cette étude les résultats de 312 jours de consommation, ces observations portent sur une période de 13 nycthémères consécutifs pour chacun des animaux.

\section{Résultats expérimentaux.}

a) Répartition temporelle de la consommation d'aliment solide et valeurs des divers paramètres. - L'enregistrement des consommations a fait apparaître pour tous les animaux une répartition inégale de la prise de nourriture au cours des deux parties du nycthémère. La partie correspondant à la plus forte ingestion d'aliment solide n'étant pas la même pour tous les animaux, nous avons distingué 2 groupes d'Oies :

- Les Oies à tendance diurne, qui ingèrent le maximum de nourriture au cours de la période d'éclairement (plus de 50 p. 100 de leur consommation quotidienne), représentent 79 p. 100 des animaux étudiés.

- Les Oies à tendance nocturne, ingérant le maximum de nourriture au cours de la 
période obscure (plus de 50 p. 100 de leur consommation quotidienne), représentent 21 p. 100 des animaux étudiés.

En moyenne, l'Oie à tendance diurne consomme 60 p. 100 de sa nourriture quotidienne dans la période éclairée $(t=6,40, P<0,001)$, alors que l'Oie à tendance nocturne en ingère $57 \mathrm{p}$. 100 pendant la période obscure $(t=5,72, P<0,001)$.

Nous avons été amenés à tenir compte de ces 2 groupes dans l'analyse effectuée.

L'étude de l'évolution des quantités ingérées, par période de $2 \mathrm{~h}$, montre une répartition inégale des prises alimentaires au cours des $24 \mathrm{~h}$. Chez tous les animaux à tendance diurne un pic de consommation apparaît dans les $2 \mathrm{~h}$ qui précèdent l'extinction des lumières. Ce pic est moins marqué chez l'Oie à tendance nocturne, mais il se retrouve par contre dans les 2 h qui précèdent l'éclairement du local. La figure 3 illustre
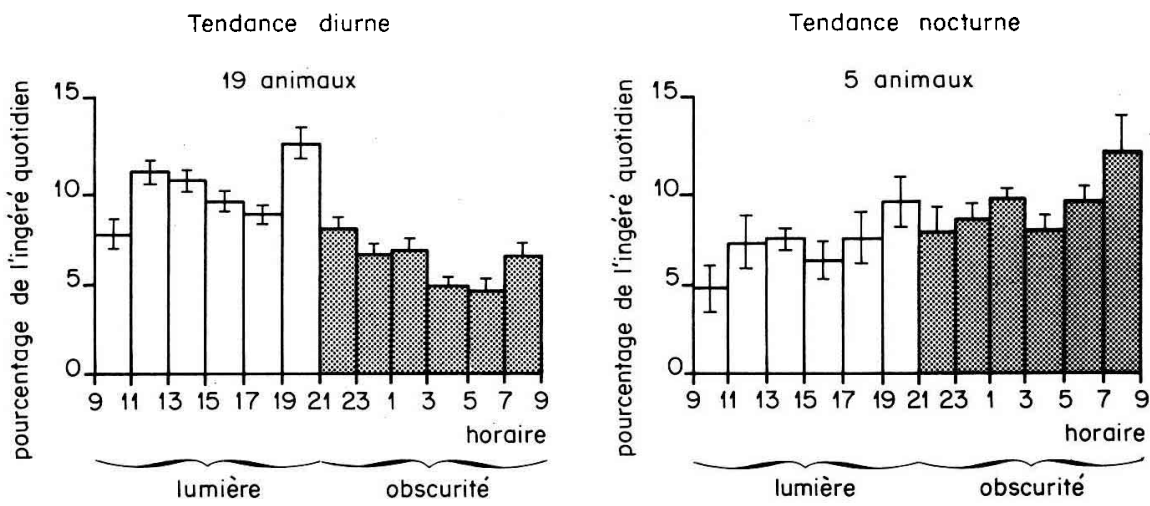

FIG. 3. - Evolution des quantités ingérées au cours du nycthémère (les écarts-types de la moyenne sont représentés).

cette évolution de l'ingéré alimentaire au cours des $24 \mathrm{~h}$ el met en évidence les heures de consommation des deux groupes d'animaux.

Nous retrouvons d'autre part, comme chez le Rat, une répétition d'un jour à l'autre du comportement alimentaire. C'est ainsi que les périodes horaires de forte consommation sont superposables d'un nycthémère à l'autre. Quelques différences apparaissent cependant entre les profils des divers individus quant à la reproduction des heures d'ingestion maximum. Ces différences sont surtoul marquées si l'on compare deux individus à tendance diurne et noclurne. L'examen des enregistrements des prises alimentaires reproduits sur la figure 4 montre que les phases de consommation sont continues, bien délimitées et séparées par des intervalles plus ou moins longs.

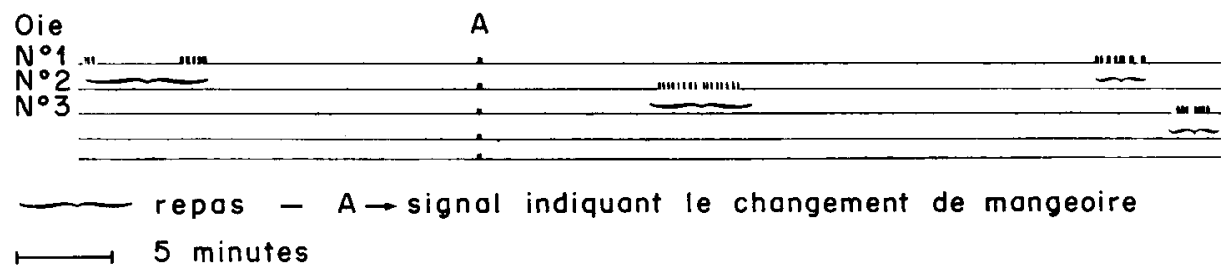

FIG. 4. - Enregistrement graphique de la séquence alimentaire de l'Oie. 
- Valeurs des paramètres apportés par le dispositif no 1.

Suite à l'analyse des données, issues des 312 enregistrements quotidiens, nous pouvons indiquer une valeur moyenne des différents paramètres caractérisant le comportement alimentaire.

Les animaux consomment $195 \pm 10 \mathrm{~g} /$ jour, ce qui représente $17,5 \pm 0,9$ repas d'une durée de $111 \pm 10 \mathrm{sec}$ et séparés par des intervalles de $75 \pm 4 \mathrm{~min}$. L'Oie n'effectue pas le même nombre de repas au cours des parties éclairée et obscure. Nous notons toujours une corrélation positive entre le nombre de repas et les quantités ingérées au cours de chacune de ces deux périodes. D'un nycthémère à l'autre, nous observons également une relation positive entre les consommations globales quotidiennes et le nombre de repas journaliers $(r=0,41, P<0,05)$.

- Valeurs des paramètres apportés par le dispositif $n^{0} 2$.

Les valeurs des ingérés par repas ainsi que celles des vitesses d'ingestion ont été déterminées sur 4 animaux mâles à tendance diurne. La taille moyenne des repas esł de $9,5 \pm 0,8 \mathrm{~g}$ avec une valeur supérieure pendant le jour ; l'ingestion se déroule à la vitesse moyenne de $11,3 \pm 2,7 \mathrm{~g} / \mathrm{min}$, cette valeur étant également supérieure pendant la partie éclairée. La différence de consommation observée entre la période éclairée et la période obscure est donc la résultante d'une augmentation des valeurs de la fréquence des repas, de l'amplitude de chacun d'eux et de la vitesse à laquelle ils se déroulent. Il ressort de l'étude statistique (test $t$ ) que les différents paramètres ne diffèrent pas significativement suivant les sexes. Par contre, des différences significatives observées entre les animaux à tendance diurne et à tendance nocturne confirment l'intérêt qu'il y a à distinguer ces deux groupes d'Oies. Le tableau 1 présente les valeurs moyennes des paramètres essentiels caractérisant le comportement alimentaire de l'Oie.

b) Relation entre les durées des intervalles pré et postprandiaux et les quantités consommées au cours des repas. - Afin de tenter de dégager des informations sur les mécanismes de déclenchement du repas, nous avons recherché l'existence d'une relation entre la quantité d'aliment consommé au cours du repas et la durée de l'intervalle qui suit ou précède celui-ci. Il a en effet été observé chez diverses espèces que la durée d'inhibition de consommation postprandiale est proportionnelle à l'importance de la prise alimentaire au cours du repas précédent (Le Magnen ef Tallon, 1966 ; Thomas ef Mayer, 1968 ; Balagura et Coscina, 1968 ; Snowdon, 1969 ; De Castro, 1978 chez le Rat ; Sanderson et Vanderweele, $1975 \mathrm{chez}$ le Lapin ; Ardisson et al., 1975, chez le Chien ; Duncan et al., 1970 chez la Poule). Cette corrélation postprandiale n'a cependant pas été observée chez le Chat (Mugford, 1977) ni chez le Cobaye (Hirsch, 1973) et a même été contestée chez le Rat (Panksepp, 1973).

Devant cette discordance entre les auteurs, nous nous sommes demandé si une telle relation se retrouvait chez l'Oie. Dans ce but, nous avons analysé 920 séquences alimentaires sur quatre animaux mâles à tendance diurne. Les quantiłés ingérées au cours de chacun de ces repas ont été couplées avec les durées des intervalles qui suivent ou précèdent chacun d'entre eux. Nous avons exclu les repas de transition entre les périodes éclairée et obscure ; les intervalles les séparant ne se plaçant pas entièrement dans l'une ou l'autre de ces parties ne peuvent en effet être pris en considération. Les données concernant chaque individu ont été analysées jour après jour et globalement sur 13 nycthémères. Les périodes diurne et nocturne ont également été distin- 


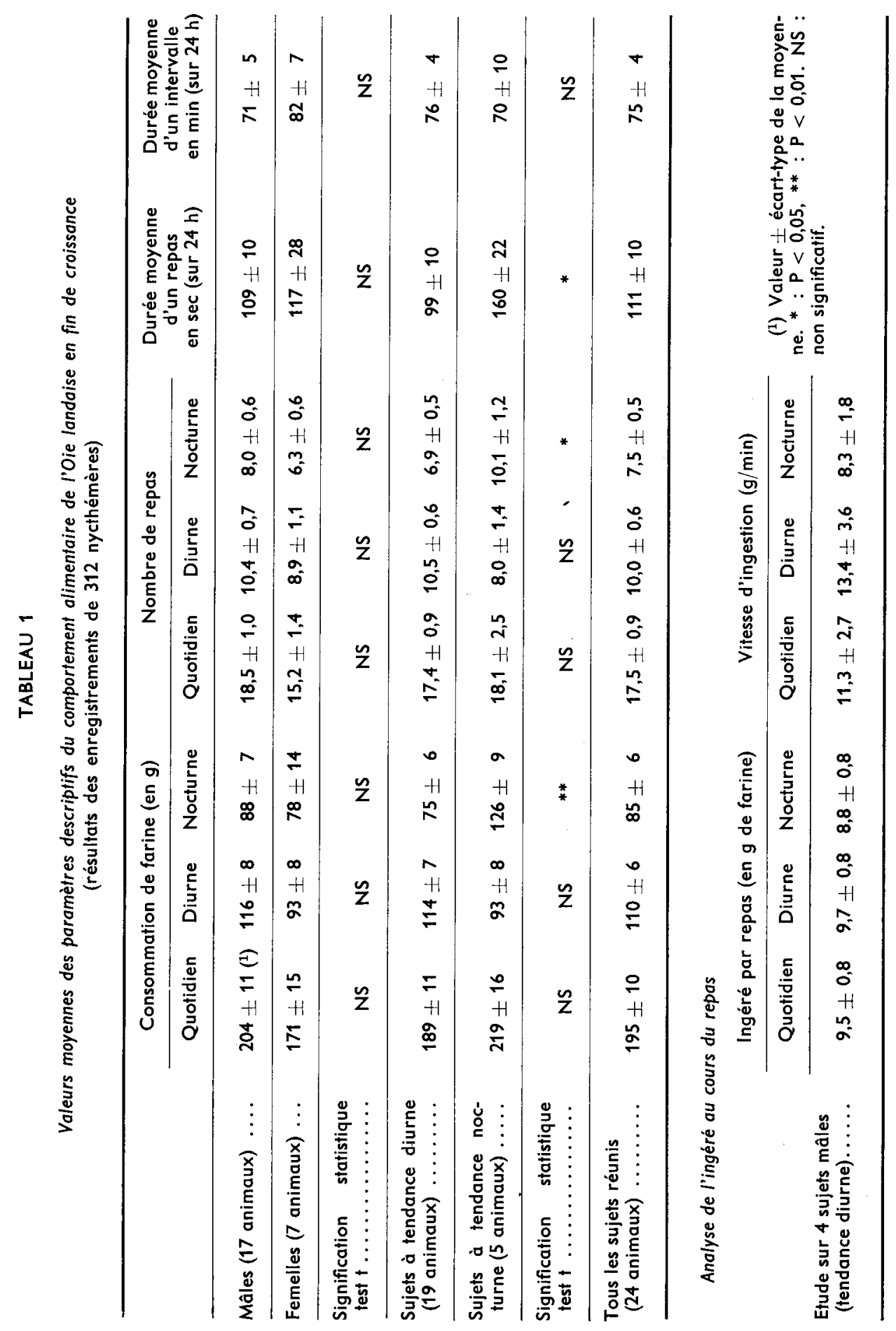


guées. Nous avons utilisé deux procédés de calcul : soit sans tenir compte de la durée du repas, soit en la faisant intervenir dans l'analyse. Dans le cas de l'Oie, l'examen des résultats individuels ef quotidiens montre une grande variabilité de réponse : en groupant pour chaque individu treize jours d'observation, nous constatons que pour la moitié d'entre eux la corrélation la plus forte est la relation avec la durée de l'intervalle après le repas, alors que pour l'autre moitié, la corrélation la plus élevée est la

TABLEAU 2

Corrélations entre les quantités d'aliment ingéré par repas et les durées des intervalles préet postprandiaux (Ełude faite avec 5 durées différentes de l'intervalle entre deux prises alimentaires.

\begin{tabular}{|c|c|c|c|c|c|c|c|c|c|}
\hline \multirow{4}{*}{ No Oie } & \multicolumn{5}{|c|}{$\begin{array}{c}\text { Corrélation entre taille du repas et durée } \\
\text { de l'intervalle pré-prandial }\end{array}$} & \multirow{2}{*}{\multicolumn{4}{|c|}{$\begin{array}{c}\begin{array}{c}\text { Corrélation entre taille du repas } \\
\text { et durée de l'intervalle } \\
\text { post-prandial }\end{array} \\
\text { corrélation }\end{array}$}} \\
\hline & \multirow{3}{*}{$\begin{array}{l}\text { Durée de } \\
\text { l'intervalle } \\
\text { entre deux } \\
\text { prises }\end{array}$} & \multicolumn{4}{|c|}{ corrélation } & & & & \\
\hline & & \multicolumn{2}{|c|}{ diurne } & \multicolumn{2}{|c|}{ nocturne } & \multicolumn{2}{|c|}{ diurne } & \multicolumn{2}{|c|}{ nocturne } \\
\hline & & $\mathbf{r}$ & $\mathbf{s}$ & $\mathbf{r}$ & $s$ & $r$ & $\mathbf{s}$ & $r$ & $\mathbf{s}$ \\
\hline \multirow{5}{*}{4003} & $7 \mathrm{~min} 30 \mathrm{sec}$ & 0,03 & NS & 0,07 & NS & 0,32 & $* *$ & 0,11 & NS \\
\hline & $15 \mathrm{~min}$ & 0,001 & NS & 0,06 & NS & 0,34 & $* *$ & 0,18 & NS \\
\hline & $20 \mathrm{~min}$ & 0,04 & NS & 0,06 & NS & 0,49 & $* *$ & 0,16 & NS \\
\hline & $30 \mathrm{~min}$ & 0,19 & NS & 0,08 & NS & 0,50 & $* *$ & 0,15 & NS \\
\hline & $45 \mathrm{~min}$ & 0,19 & NS & 0,24 & NS & 0,22 & NS & 0,15 & NS \\
\hline \multirow{5}{*}{47} & $7 \mathrm{~min} 30 \mathrm{sec}$ & 0,46 & $* *$ & 0,42 & $* *$ & 0,18 & NS & 0,17 & NS \\
\hline & $15 \mathrm{~min}$ & 0,38 & $* *$ & 0,40 & $* *$ & 0,08 & NS & 0,22 & NS \\
\hline & $20 \mathrm{~min}$ & 0,33 & $* *$ & 0,21 & NS & 0,09 & NS & 0,23 & $*$ \\
\hline & $30 \mathrm{~min}$ & 0,02 & NS & 0,32 & * & 0,01 & NS & 0,43 & $* *$ \\
\hline & $45 \mathrm{~min}$ & 0,08 & NS & 0,16 & NS & 0,23 & NS & 0,38 & $* * *$ \\
\hline \multirow{5}{*}{4109} & $7 \mathrm{~min} 30 \mathrm{sec}$ & 0,29 & $* *$ & 0,15 & $*$ & 0,06 & NS & 0,05 & NS \\
\hline & $15 \mathrm{~min}$ & 0,26 & $* *$ & 0,21 & $*$ & 0,16 & NS & 0,21 & $*$ \\
\hline & $20 \mathrm{~min}$ & 0,04 & NS & 0,49 & $* *$ & 0,17 & NS & 0,03 & NS \\
\hline & $30 \mathrm{~min}$ & 0,09 & NS & 0,52 & $* *$ & 0,63 & $* *$ & 0,47 & $* *$ \\
\hline & $45 \mathrm{~min}$ & 0,30 & NS & 0,34 & NS & 0,88 & $* * *$ & 0,37 & $*$ \\
\hline \multirow{5}{*}{1815} & $7 \mathrm{~min} 30 \mathrm{sec}$ & 0,15 & NS & 0,04 & NS & 0,23 & $* *$ & 0,15 & NS \\
\hline & $15 \mathrm{~min}$ & 0,04 & NS & 0,10 & NS & 0,11 & NS & 0,03 & NS \\
\hline & $20 \mathrm{~min}$ & 0,02 & NS & 0,13 & NS & 0,25 & $* *$ & 0,02 & NS \\
\hline & $30 \mathrm{~min}$ & 0,003 & NS & 0,24 & NS & 0,49 & $* *$ & 0,11 & NS \\
\hline & $45 \mathrm{~min}$ & 0,05 & NS & 0,28 & NS & 0,69 & $* * *$ & 0,13 & NS \\
\hline
\end{tabular}

$\mathbf{r}=$ coefficient de corrélation ; $\mathbf{s}=$ signification.

* : $\mathrm{P}<0,05$, **: $\mathrm{P}<0,01$, ***: $\mathrm{P}<0,001$. 
relation avec la durée de l'intervalle précédent. Le fait de prendre en compte la durée du repas ne modifie pas significativement les valeurs des coefficients de corrélation.

Les désaccords entre les auteurs, concernant l'existence de ces corrélations, nous ont conduit à rechercher si la variation des réponses ne résidait pas dans le choix de la définition du repas. C'est ainsi que nous avons envisagé plusieurs possibilités qui ont été adoptées chez le Rat par ces auteurs et considéré deux repas comme distincts lorsqu'ils étaient séparés par un intervalle de 15, 20, 30 ou 45 min.

Le tableau 2 fournit les différentes valeurs du coefficient de corrélation en fonction du critère choisi pour définir le repas (lors de la mesure de la durée des intervalles, nous avons ajouté la durée des repas qui les précèdent).

Lorsque nous nous rapprochons du critère adopté pour le Rał (intervalle entre deux repas : $40 \mathrm{~min}$ ), la corrélation : ingéré par repas-durée de l'intervalle suivant, est significativement positive, alors que la relation préprandiale, qui existe chez la moitié des Oies éfudiées avec noire premier critère, disparaît. Ceffe étude illustre parfaitement l'importance de la définition du repas, les résultats pouvant être contradictoires suivant le choix qui a été effectué.

\section{Conclusion. Discussion.}

L'Oie manifeste une double périodicité de sa séquence alimentaire : répétition de repas bien individualisés suivis d'intervalles plus ou moins longs, et une périodicité journalière, l'animal ingérant le maximum de nourriture à certaines heures caractéristiques du nycthémère pour chacun des sujets.

L'Oie présente une différence relativement faible entre les valeurs de ses consommations en période éclairée et période obscure. Ceci peut laisser supposer que les mécanismes de la régulation lipostatique, montrés par Le Magnen ef al. (1975) chez le Rat, qui président au cycle jour-nuit de cet animal, ne sonł peut-être pas applicables à l'Oie. On peut toutefois penser qu'il y a bien alternance des états de lipogénèse et de lipolyse qui correspondent respectivement aux périodes d'activité alimentaire ef de repos mais que ces phases sont alors moins différenciées chez l'Oie. On ne peut parler en effet, chez cet animal, d'une réelle période de repos alimentaire mais plutôt d'une diminution du niveau des consommations qui se situe soit de jour soit de nuit suivant les individus. L'analyse nous a permis de distinguer deux grands groupes d'animaux d'après les heures où ceux-ci placent le maximum de leur activité alimentaire. On peut admettre que l'existence de ces groupes est une des caractéristiques de l'espèce. Toutefois nous pouvons également émettre l'hypothèse selon laquelle certaines habitudes alimentaires seraient acquises dans la période de préexpérimentation. Les animaux au cours de cette période sont en effet élevés en troupeau et donc soumis à une hiérarchie sociale.

A l'intérieur de ces deux groupes les animaux ont un profil alimentaire assez semblable d'un individu à l'autre et d'un jour à l'autre. Nos observations relatives à la mise en évidence d'un pic de consommation dans les $2 \mathrm{~h}$ qui précèdent la période obscure, chez les animaux à tendance diurne, sont bien en accord avec les résultats rapportés par Weaver et Siegel (1968), Centa et al. (1969) puis par Goussopoulos et al. (1973) qui signalent une activité alimentaire accrue au cours des heures qui précèdent l'extinction des lumières chez le Poulet. Par contre chez l'Oie le $2^{\mathrm{e}}$ pic de consommation 
ne se situe pas comme chez le Poulet après l'allumage des lumières, mais précède la période éclairée. Le fait même que toutes les Oies ne présentent pas un profil alimentaire identique prouve que les individus ne sont conditionnés ni par le dispositif d'enregistrement ni par une éventuelle influence réciproque des animaux. Aucune différence significative, quant au déroulement de la séquence alimentaire n'est notée entre mâles et femelles, mais nous devons émettre une certaine réserve du fait de l'inégaliłé, constatée a posteriori, dans la répartition des sexes des Oies utilisées ( 17 mâles ef 7 femelles).

L'analyse statistique que nous avons réalisée ne nous permet pas d'affirmer qu'il existe une corrélation entre la quantité d'aliment consommée au cours du repas et la durée des intervalles qui le suivent ou le précèdent. II est de toute évidence que le choix de la définition du repas influence les résultats obtenus au cours de cette étude.

Les désaccords constatés entre les auteurs, que nous avons signalés, pourraient partiellement s'expliquer par le fait que le mode de calcul utilisé pour étudier les relations entre intervalle et quantité consommée au cours d'un repas n'est pas le même pour tous. D'après Le Magnen et Devos (1980), il semble préférable de prendre en compte la durée du repas avec la durée de l'intervalle qui suit ou précède. On peut en effet penser que les mécanismes qui contrôlent l'initiation d'un repas se mettent en place dès le début du repas qui précède. Chez le Rat, le coefficient de corrélation postprandiale nocturne ainsi calculé a une valeur plus élevée que celle obtenue lors des études antérieures.

L'Oie présente un profil alimentaire peu favorable à ce genre d'étude car l'existence d'une corrélation significative ou non dépend en fait du critère repas choisi. Ce type d'analyse semble plus facilement envisageable chez les espèces effectuant des repas peu nombreux et très bien individualisés.

Reçu en février 1981. Accepté en avril 1981.

\section{Références}

ARDISSON J. L., DOLISI C., CAMOUS J. P., OZON C., 1975. Influence of eating modalities on water intake in dog. J. Physiol., Paris. 70, 139-148.

AUFFRAY P., BLUM J. C., 1970. Hyperphagie et stéatose hépatique chez l'Oie après lésion du noyau ventro-médian de l'hypothalamus. C.R. Acad. Sci. Paris, Sér. D, 270, 2362-2365.

AUfFraY P., MARCILLOUX J. C., BAHY C., ALBE-FESSARD D., 1973. Hyperphagie induite chez l'oie par injections intraventriculaires de 6-hydroxydopamine. C. R. Acad. Sci. Paris, Sér. D, 276, 347-350.

BALAGURA S., COSCINA D. V., 1968. Periodicity of food intake in the rat as measured by an operant response. Physiol. Behav., 3, 641-643.

CENTA D. M., FOSHEE D. P., HOWES J. R., 1969. Control of feeding behaviour in poultry. Physiol. Rep., 24, 153-154.

CLIFTON P. G., 1979. Temporal patterns of feeding in the domestic chick. I. ad libitum. Anim. Behav., 27, 811-820.

DE CASTRO J. M., 1978. An analysis of the variance in meal patterning. Neurosci. biobehav. Rev., 2, 301-310.

DUNCAN I. J. H., HORNE A. R., HUGHES B. O., 1970. The pattern of food intake in female brown Leghorn fowls as recorded in a skinner box. Anim. Behav., 18, 245-255.

GOUSSOPOULOS J., CARLES Y., PRUD'HON M., BACOU F., 1973. Enregistrement graphique de l'activité ef du comportement alimentaire du poulet. Ann. Zootech., 22, 133-145. 
HIRSCH E., 1973. Some deferminants of intake and patterns of feeding in the guinea-pigs. Physiol. Behov., 11, 687-704.

LE MAGNEN J., DEVOS M., 1980. Parameters of the meal pattern in rats : their assessment and physiological significance. Neurosci. biobehav. Rev., Suppl. 1, 1-11.

LE MAGNEN J., DEVOS M., GAUDILLERE J. P., LOUIS-SYLVESTRE J., TALLON S., 1973. Role of the lipostatic mechanism in regulation by feeding of energy balance. J. comp. physiol. Psychol., 84, 1-23.

LE MAGNEN J., TALLON S., 1966. La périodicité spontanée de la prise d'aliments ad libitum du rat blanc. J. Physiol. Paris, 58, 323-349.

MUGFORD R. A., 1977. External influences on the feeding of carnivores, 25-50. In KARE M. R., MALLER O., Chemical senses and nutrition. Acad. Press, N. Y.

PANKSEPP J., 1973. Reanalysis of feeding patterns in the rat. J. comp. physiol. Psychol., 82, 78-94.

SANDERSON J. D., VANDERWEELE D. A., 1975. Analysis of feeding pattern in normal and vagotomized rabbits. Physiol. Behav., 15, 357-364.

SNEDECOR G. W., COCHRAN W. G., 1971. Méthodes statistiques. ACTA, Paris, 6e éd.

SNOWDON C. T., 1969. Motivation regulation and the control of meal parameters with oral and intragastric feeding. J. comp. physiol. Psychol., 69, 91-100.

THOMAS D. W., MAYER J., 1968. Meal taking and regulation of food intake by normal and hypothalamic hyperphagie rats. J. comp. physiol. Psychol., 66, 642-653.

VAN HEMEL S. B., MYER J. S., 1969. Feeding patterns and response to caloric dilution in the Japanese quail. Physiol. Behav., 4, 339-344.

WEAVER W. D. Jr., SIEGEL P. B., 1968. Photoperiodism as a factor in feeding rhythms of broiler chickens. Poult. Sci., 47, 1148-1154.

ZEIGLER H. P., GREEN H. L., LEHRER R., 1971. Patterns of feeding behavior in the pigeon. J. Physiol. Psychol., 76, 468-477. 